\title{
Novel Fluorescent Fluorine-Boron Complexes: Synthesis, Crystal Structure, Photoluminescence and Electrochemistry properties
}

\author{
Ying Zhou, ${ }^{\dagger}{ }^{\text {Yi Xiao }}{ }^{\dagger, ~ * ~ D a n ~ L i, ~}{ }^{\dagger}$ Meiyan Fu, ${ }^{\dagger,}$ Xuhong Qian, ${ }^{\ddagger}$, * \\ State Key Laboratory of Fine Chemicals, Dalian University of Technology, Dalian 116012, China \\ Virtual Laboratory of Computational chemistry, CNIC Chinese Academy of Science \\ Shanghai Key Laboratory of Chemical Biology, East China University of Science and Technology, \\ Shanghai 200237, China
}

xiaoyi@chem.dlut.edu.cn, xhqian@ecust.edu.cn.

Page S1 Contents

S2 General experimental procedures

Synthesis of compounds 3 and 4

S3 Table S1. Geometrical parameters of hydrogen bond $\left(\AA{ }^{\circ}\right)$, involved in the molecular construction in 1 and 2

Table S2. Arene - arene packing geometries ( $\left.\AA{ }^{\circ}\right)$, Involved in crystal 1 and 2

Figure S1. The views of hydrogen-bonded chains aligned parallel to each other by $\pi-\pi$ stacking in crystal 1

S4 Figure S2. View of the assembled structures, showing the C-H $\cdots$ F-B and $\mathrm{C}-\mathrm{H}^{\cdots} \mathrm{O}$ intermolecular interactions in crystal 2

Table S3. Selected bond lengths $(\AA)$ and angles $\left({ }^{\circ}\right)$ for crystals 1 and 2

S5 Table S4. Crystallographic Data for crystals 1 and 2

Figure S3. Absorption spectra of compound 1 in different solvents

S6 Figure S4. Absorption spectra of compound 2 in different solvents

Figure S5. Fluorescent emission spectra of compound 1 in different solvents

Figure S6 Fluorescent emission spectra of compound 2 in different solvents

S7 Table S5. Photophysical Characteristics of compounds 1 and 2 in different solvents

Figure S7. The picture of solid fluorescence of BODIPY derivative, which was taken under a handheld UV (365 $\mathrm{nm}$ ) lamp.

Figure S8. The molecular structure of BODIPY used in CV for comparison in present work.

S8 Figure S9. The LUMO and HOMO levels of TM-BODIPY calculated by semiempirical calculations.

${ }^{1} \mathrm{H}-\mathrm{NMR}$ Spectra of compound 1

S9 ${ }^{13} \mathrm{C}$ NMR for compound 1

${ }^{1} \mathrm{H}$ NMR for compound 2

$\mathrm{S} 10{ }^{13} \mathrm{C}$ NMR for compound 2

Symbolic Z-matrix for compound 1

S12 Symbolic Z-matrix for compound 2 
Electrochemical Measurements: Electrochemical studies made use of cyclic voltammetry with a conventional 3electrode system using a $100 \mathrm{~W}$ electrochemical analyzer in deoxygenated and anhydrous acetonitrile at room temperature. The potentials are reported vs ferrocene as internal standard using a scan rate of $100 \mathrm{~m} \mathrm{Vs}^{-1}$, glassy carbon working electrode, $\mathrm{Ag} / \mathrm{AgNO}_{3}$ reference electrode, platinum counter electrode, and the sample solutions contained $1.0 \times 10^{-3} \mathrm{M}$ sample and $0.1 \mathrm{M}$ tetrabutylammonium hexafluorophosphate as a supporting electrolyte. Argon was bubbled for 10min before each measurement.

Experimental procedure for X-ray crystallographic analysis: Crystals were obtained by direct diffusion of aether into a solution of $\mathbf{1}$ and $\mathbf{2}$ in dichloromethane, respectively. A suitable single crystal was mounted in a glass fiber, and diffraction measurements were taken with a diffractometer with Mo $\mathrm{K} \alpha$ graphite monochromated radiation. The structures were solved by direct methods using the programme SHELXL-97. The refinement and all further calculations were carried out using SHELXL-97. The non-H atoms were refined anisotropically, using weighted fullmatrix least-squares on $\mathrm{F}^{2}$. Absorption correction were applied based on multiple and symmetry-equivalent measurements. The hydrogen atoms were included in the models in calculated positions and were refined as constrained to bonding atoms.

Computational Data: Molecular orbital calculations for these compounds on the density-functional theory (DFT) level using a (B3LYP/6-31G) basis set, employing the Gaussian 03 suit of programs. Geometric parameters from X-ray diffraction analysis were used for the calculations. The total energy of compound 1 is -948.1485 (a.u.) and that of compound 2 is -1101.7578 (a.u.). The Z-matrixes of these compounds are listed at the end of this supporting information.

Synthesis of Compound 3: $10.0 \mathrm{~g}$ isobenzofuran-1,3-dione $(67.5 \mathrm{mmol})$ and $10.41 \mathrm{~g}$ acetamine (135 mmol) were added in $20 \mathrm{ml}$ acetic acid, and the reaction mixture was then poured into water after it refluxed for 1 hour. The white needles collected by filtration were washed by water and dried in vacuo. Yield: $8.24 \mathrm{~g}(83.1 \%)$. The products were used without further purifications.

Synthesis of Coupound 4: $2.0 \mathrm{~g}$ compound 3 (13.6 mmol) and $3.88 \mathrm{~g}$ Sn (2.4 equiv.) were added in the mixture of 10 $\mathrm{ml}$ acetic acid and $5 \mathrm{ml} \mathrm{HCl}$. The reaction mixture was filtrated without cooling after it refluxed for 2 hours. After removal of acetic acid from the concentration in vacuo, the resulting filtrate was extracted with dichloromethane $(3 \times 50$ $\mathrm{ml})$. The organic extracts were washed with water and brine, dried over anhydrous $\mathrm{MgSO} 4$, and concentrated in vacuo. The residue was purified by silica gel column chromatography using n-hexane / ethyl acetate (1:3, v/v) as eluent to afford light-yellow powder compound $4 .{ }^{[1]}$ Yield: $0.9 \mathrm{~g}(50 \%)$, mp: $149-151{ }^{\circ} \mathrm{C} .\left(\right.$ (lit. ${ }^{[2]} \mathrm{mp} 150-151{ }^{\circ} \mathrm{C}$ ). 
Table S1. Geometrical parameters of hydrogen bond $\left(\AA \mathrm{e}^{\circ}\right)$, involved in the molecular construction in $\mathbf{1}$ and $\mathbf{2}$

\begin{tabular}{|c|c|c|c|c|}
\hline Compound & D-H ${ }^{\cdots A} A$ & $\mathbf{d}(\mathbf{H} \cdots \mathbf{A})$ & $\mathbf{d}\left(\mathbf{D}^{\cdots} \mathbf{A}\right)$ & (DHA) \\
\hline \multirow[t]{3}{*}{1} & (a) $\mathrm{C}(6)-\mathrm{H}(6 \mathrm{~B}) \cdots \mathrm{F}(1 \mathrm{C})$ & 2.53 & 3.44 & 165.36 \\
\hline & (b) $\mathrm{C}(9)-\mathrm{H}(9 \mathrm{~B}) \cdots \mathrm{F}(1 \mathrm{C})$ & 2.49 & 3.37 & 159.37 \\
\hline & (c) $\mathrm{C}(4)-\mathrm{H}(4 \mathrm{~B}) \cdots \mathrm{F}(2 \mathrm{C})$ & 2.49 & 3.32 & 149.68 \\
\hline \multirow[t]{2}{*}{2} & (d) $\mathrm{C}(8)-\mathrm{H}(8 \mathrm{~A}) \cdots \mathrm{F}(1)$ & 2.53 & 3.41 & 158.45 \\
\hline & (e) $\mathrm{C}(7)-\mathrm{H}(7 \mathrm{~A}) \cdots \mathrm{O}(1)$ & 2.51 & 3.34 & 148.54 \\
\hline \multicolumn{5}{|c|}{$\begin{array}{l}\mathrm{D}=\text { donor; } \mathrm{A}=\text { acceptor } \\
\text { Symmetry codes: } \\
\qquad \text { (a) , (b) and (c) }=-1 / 2+\mathrm{x}, 1 / 2-\mathrm{y},-1 / 2+\mathrm{z} \\
\text { (d) }=\mathrm{x}, 1 / 2-\mathrm{y},-1 / 2+\mathrm{z} ;(\mathrm{e})=-1+\mathrm{x}, 1 / 2-\mathrm{y},-1 / 2+\mathrm{z}\end{array}$} \\
\hline
\end{tabular}

Table S2. Arene - arene packing geometries $\left(\AA{ }^{\circ}{ }^{\circ}\right)$, involved in crystal $\mathbf{1}$ and $\mathbf{2}$

\begin{tabular}{cccccc}
\hline & & & & \\
& Interplanar & & & \\
Compound & distance & angle & $\mathrm{d}^{\mathrm{a}}$ & $\mathrm{Hd}^{\mathrm{b}}$ & $\theta^{\mathrm{c}}$ \\
\hline $\mathbf{1}$ & (a) 3.440 & 0.0 & $3.895^{\mathrm{d}, \mathrm{e}}$ & 1.827 & 27.98 \\
\cline { 2 - 6 } & (b) 3.362 & 4.6 & $3.608^{\mathrm{f}, \mathrm{g}}$ & 1.309 & 21.27 \\
\hline & (a) 3.580 & 5.5 & $3.970^{\mathrm{h}, \mathrm{i}}$ & 1.715 & 25.60
\end{tabular}

${ }^{a}$ Ring centroid to ring centroid distance; ${ }^{b}$ Horizontal displacement between the two ring centroid; ${ }^{c}$ Ring normal to vector between the ring centroids angle. ${ }^{\mathrm{d}}$ Centroid for N2D-C1D to C5D, ${ }^{\mathrm{e}}$ Centroid for N2B-C1B to C5B; ${ }^{\mathrm{f}}$ Centroid for N2F-C1F to C5F; ${ }^{g}$ Centroid for C8E to C13E; ${ }^{\text {h }}$ Centroid for C1A to C6A, ${ }^{\mathrm{i}}$ Centroid for $\mathrm{C} 12 \mathrm{~A}$ to $\mathrm{C} 17 \mathrm{~A}$.

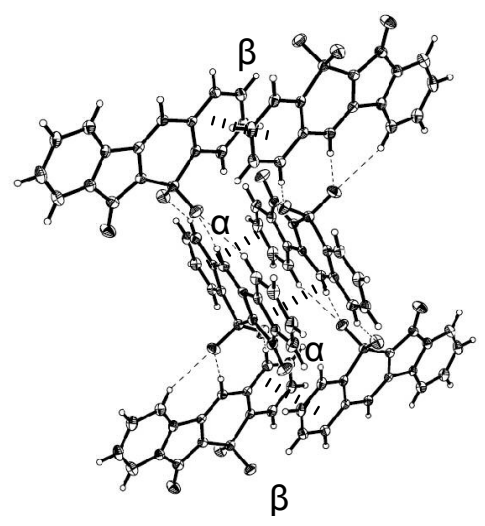

Figure S1. The views of hydrogen-bonded chains aligned parallel to each other by $\pi-\pi$ stacking in crystal $1(\alpha=3.44 \AA ; \beta=3.36 \AA)$ 


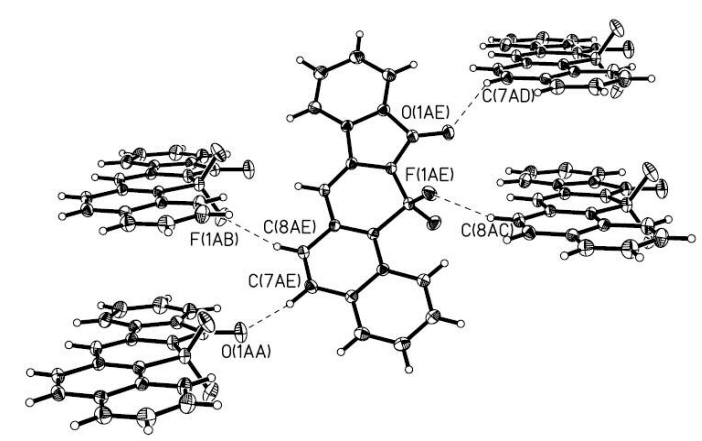

Figure S2. View of the assembled structures, showing the C-H ${ }^{\cdots}$ F-B and C-H ${ }^{\cdots} \mathrm{O}$ intermolecular interactions in crystal 2

Table S3. Selected bond lengths $(\AA)$ and angles $\left(^{\circ}\right)$ for crystals $\mathbf{1}$ and $\mathbf{2}$

\begin{tabular}{cllll}
\hline Compound & \multicolumn{3}{c}{ Bond lengths } & Bond angles \\
\hline $\mathbf{1}$ & $\mathrm{N}(1)-\mathrm{C}(14)$ & $1.397(2)$ & $\mathrm{C}(14)-\mathrm{N}(1)-\mathrm{B}(1)$ & $126.17(13)$ \\
& $\mathrm{N}(1)-\mathrm{B}(1)$ & $1.517(2)$ & $\mathrm{F}(2)-\mathrm{B}(1)-\mathrm{F}(1)$ & $109.88(13)$ \\
$\mathrm{O}(1)-\mathrm{C}(14)$ & $1.2113(19)$ & $\mathrm{F}(2)-\mathrm{B}(1)-\mathrm{N}(1)$ & $113.00(16)$ \\
$\mathrm{B}(1)-\mathrm{F}(1)$ & $1.386(2)$ & $\mathrm{F}(1)-\mathrm{B}(1)-\mathrm{N}(1)$ & $111.20(14)$ \\
& $\mathrm{B}(1)-\mathrm{F}(2)$ & $1.377(2)$ & $\mathrm{F}(2)-\mathrm{B}(1)-\mathrm{N}(2)$ & $107.88(13)$ \\
$\mathrm{B}(1)-\mathrm{N}(2)$ & $1.594(2)$ & $\mathrm{F}(1)-\mathrm{B}(1)-\mathrm{N}(2)$ & $106.66(15)$ \\
& $\mathrm{C}(1)-\mathrm{N}(2)$ & $1.356(2)$ & $\mathrm{N}(1)-\mathrm{B}(1)-\mathrm{N}(2)$ & $107.95(12)$ \\
$\mathrm{C}(6)-\mathrm{C}(7)$ & $1.335(2)$ & $\mathrm{C}(7)-\mathrm{C}(6)-\mathrm{C}(5)$ & $121.92(14)$ \\
& $\mathrm{F}(1)-\mathrm{B}(1)$ & $1.381(3)$ & $\mathrm{F}(1)-\mathrm{B}(1)-\mathrm{F}(2)$ & $110.28(17)$ \\
$\mathrm{F}(2)-\mathrm{B}(1)$ & $1.384(3)$ & $\mathrm{F}(1)-\mathrm{B}(1)-\mathrm{N}(1)$ & $110.93(17)$ \\
& $\mathrm{N}(1)-\mathrm{B}(1)$ & $1.508(3)$ & $\mathrm{C}(18)-\mathrm{N}(1)-\mathrm{B}(1)$ & $124.80(15)$ \\
$\mathrm{N}(2)-\mathrm{B}(1)$ & $1.611(2)$ & $\mathrm{C}(1)-\mathrm{N}(2)-\mathrm{B}(1)$ & $118.37(15)$ \\
$\mathrm{N}(2)-\mathrm{C}(9)$ & $1.352(2)$ & $\mathrm{F}(2)-\mathrm{B}(1)-\mathrm{N}(1)$ & $110.76(16)$ \\
& $\mathrm{O}(1)-\mathrm{C}(18)$ & $1.216(2)$ & $\mathrm{F}(1)-\mathrm{B}(1)-\mathrm{N}(2)$ & $107.79(15)$ \\
$\mathrm{C}(9)-\mathrm{C}(10)$ & $1.434(2)$ & $\mathrm{F}(2)-\mathrm{B}(1)-\mathrm{N}(2)$ & $108.19(16)$ \\
$\mathrm{C}(10)-\mathrm{C}(11)$ & $1.344(2)$ & $\mathrm{N}(1)-\mathrm{B}(1)-\mathrm{N}(2)$ & $108.78(15)$ \\
\hline
\end{tabular}


Table S4. Crystallographic Data for crystals 1 and 2

\begin{tabular}{|c|c|c|}
\hline & 1 & 2 \\
\hline formula & C14 H9 B F2 N2 O & C18 H11 B F2 N2 O \\
\hline formula weight & 270.04 & 320.10 \\
\hline $\mathrm{T}(\mathrm{K})$ & 273(2) & 273(2) \\
\hline crystal system & Monoclinic & Monoclinic \\
\hline space group & $\mathrm{P} 2(1) / \mathrm{n}$ & $\mathrm{P} 2(1) / \mathrm{c}$ \\
\hline $\mathrm{a}(\AA)$ & 11.4034 & 8.6750 \\
\hline $\mathrm{b}(\AA)$ & 8.9648 & 11.4882 \\
\hline c $(\AA)$ & 12.4160 & 14.4342 \\
\hline$\alpha(\operatorname{deg})$ & 90 & 90 \\
\hline$\beta(\operatorname{deg})$ & $109.830(2)$ & $101.136(3)$ \\
\hline$\gamma(\mathrm{deg})$ & 90 & 90 \\
\hline $\mathrm{Z}$ & 4 & 4 \\
\hline $\mathrm{V}\left(\AA^{3}\right)$ & $1194.01(5)$ & $1411.4(3)$ \\
\hline$D_{\text {calc }}\left(\mathrm{Mg} / \mathrm{m}^{3}\right)$ & 1.502 & 1.506 \\
\hline $\mathrm{F}(000)$ & 552 & 656 \\
\hline$\theta \operatorname{rang}\left(^{\circ}\right)$ & $2.10-25.00$ & $2.39-27.40$ \\
\hline \multirow[t]{3}{*}{ index ranges } & $-13 \leq \mathrm{h} \leq 13$ & $-10 \leq \mathrm{h} \leq 11$ \\
\hline & $-10 \leq \mathrm{k} \leq 8$ & $-14 \leq \mathrm{k} \leq 14$ \\
\hline & $-14 \leq \mathrm{l} \leq 14$ & $-18 \leq 1 \leq 15$ \\
\hline reflections collected & 4618 & 7810 \\
\hline independent reflections & $1939\left[\mathrm{R}_{\mathrm{int}}=0.0234\right]$ & $3158\left[\mathrm{R}_{\mathrm{int}}=0.0346\right]$ \\
\hline data/restraints/parameters & $1939 / 0 / 181$ & $3158 / 0 / 217$ \\
\hline goodness-of-fit on $\mathrm{F}^{2}$ & 0.995 & 0.999 \\
\hline \multirow[t]{2}{*}{ final $R$ indices $[\mathrm{I}>2 \sigma(\mathrm{I})]$} & $\mathrm{R} 1=0.0349$ & $\mathrm{R} 1=0.0446$ \\
\hline & $\mathrm{wR} 2=0.09 \quad \mathrm{~S} 5$ & $\mathrm{wR} 2=0.1025$ \\
\hline \multirow[t]{2}{*}{$\mathrm{R}$ indices (all data) } & $\mathrm{R} 1=0.045^{\circ}$ & $\mathrm{R} 1=0.0937$ \\
\hline & $\mathrm{wR} 2=0.1049$ & $\mathrm{wR} 2=0.1229$ \\
\hline Largest diff. peak/hole (e $\left.\AA^{3}\right)$ & $0.165 /-0.130$ & $0.151 /-0.183$ \\
\hline
\end{tabular}

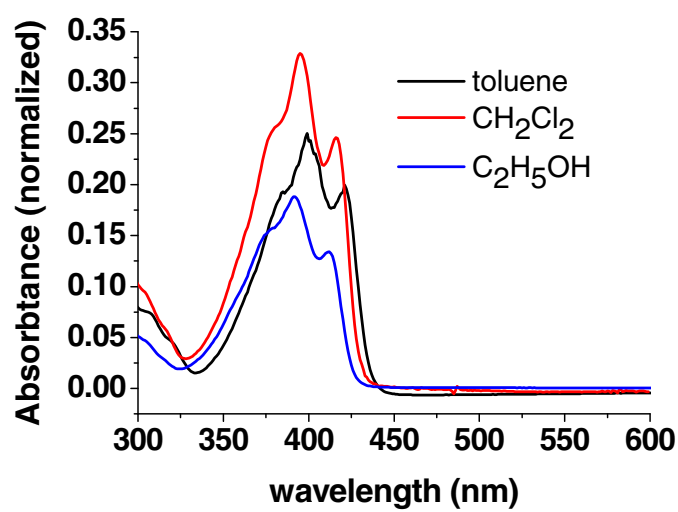

Figure S3. Absorption spectra of compound $\mathbf{1}$ in different solvents 


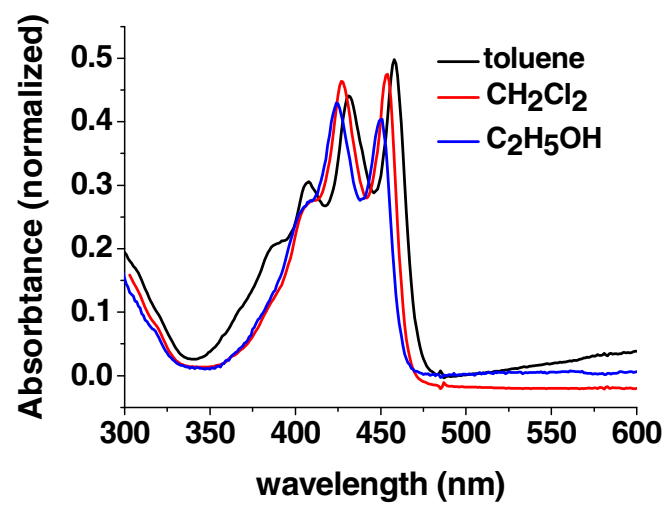

Figure S4. Absorption spectra of compound 2 in different solvents

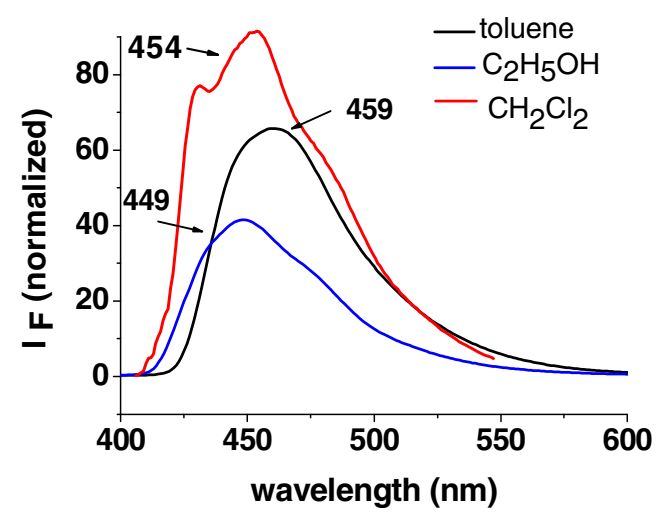

Figure S5. Fluorescent emission spectra of compound $\mathbf{1}$ in different solvents

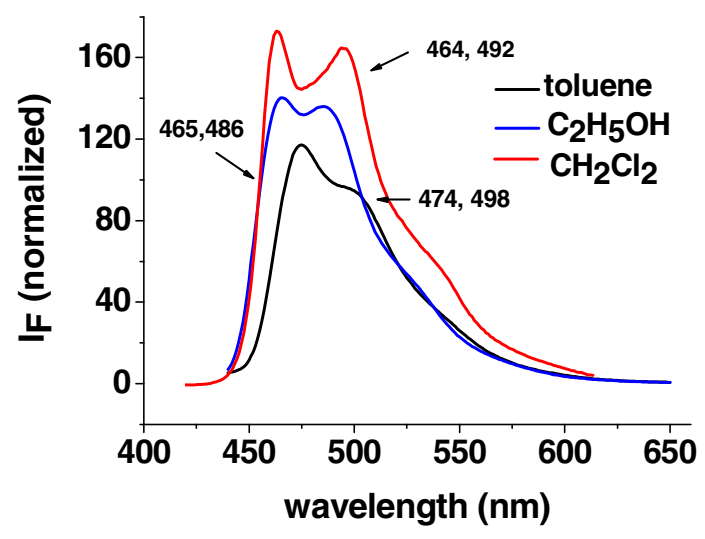

Figure S6. Fluorescent emission spectra of compound $\mathbf{2}$ in different solvents 
Table S5. Photophysical Characteristics of compounds $\mathbf{1}$ and $\mathbf{2}$ in different solvents

\begin{tabular}{ccccc}
\hline & & $\begin{array}{c}\text { Absorbtanc } \\
\text { e }\end{array}$ & Fluorescence & Stock's shift \\
compound & solvent & $\lambda$ abs $(\mathrm{nm})$ & $\lambda \mathrm{em}(\mathrm{nm})$ & $(\mathrm{nm})$ \\
\hline 1 & toluene & 399 & 459 & 60 \\
& $\mathrm{CH}_{2} \mathrm{Cl}_{2}$ & 396 & 454 & 58 \\
& $\mathrm{C}_{2} \mathrm{H}_{5} \mathrm{OH}$ & 391 & 449 & 58 \\
2 & toluene & 431,458 & 474,498 & 43 \\
& $\mathrm{CH}_{2} \mathrm{Cl}_{2}$ & 427,454 & 464,492 & 38 \\
& $\mathrm{C}_{2} \mathrm{H}_{5} \mathrm{OH}$ & 423,450 & 465,486 & 36 \\
\hline
\end{tabular}
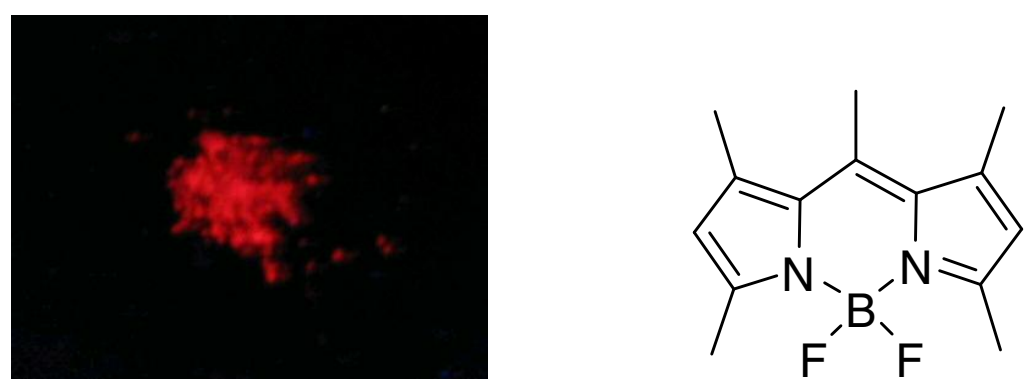

Figure S7. The picture of solid fluorescence of BODIPY derivative, which was taken under a handheld UV (365 nm) lamp.

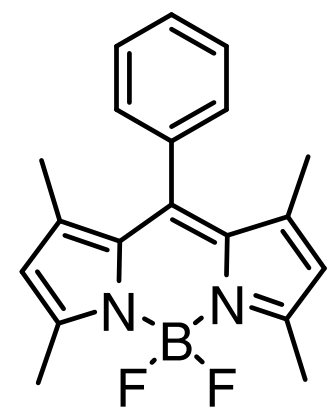

Figure S8. The molecular structure of BODIPY used in CV for comparation in present work. 

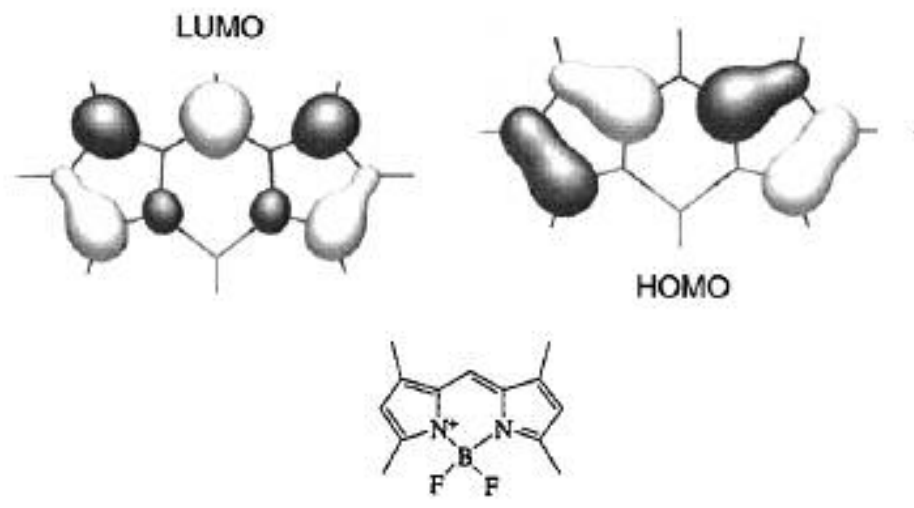

\section{TM-BODIPY $^{\circledR}$}

Figure S9. The LUMO and HOMO levels of TM-BODIPY calculated by semiempirical calculations. ${ }^{[3]}$

\section{${ }^{1}$ H-NMR Spectra of Compound 1}

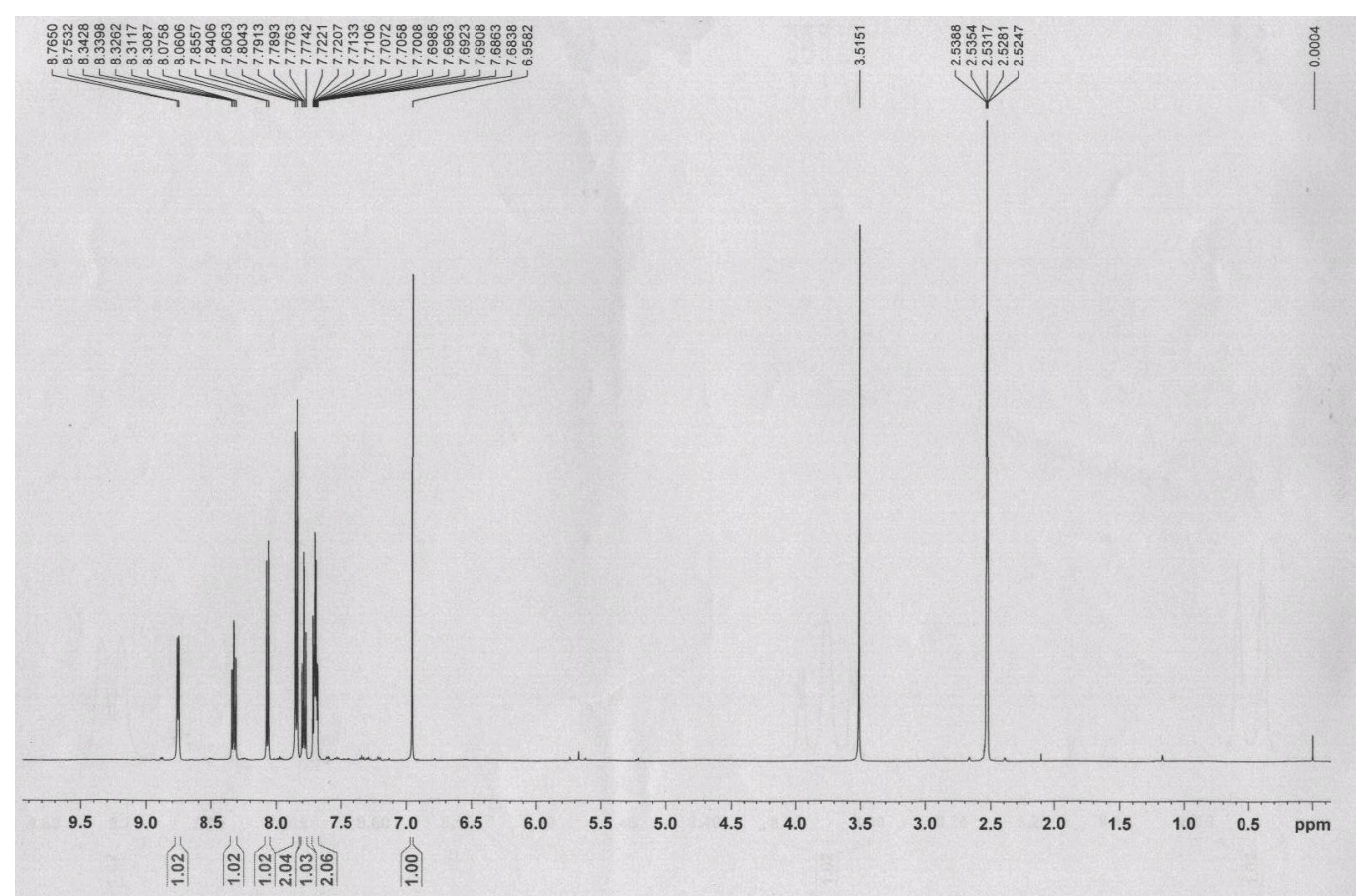




\section{${ }^{13} \mathrm{C}-\mathrm{NMR}$ spectra of Compound $\mathbf{1}$}

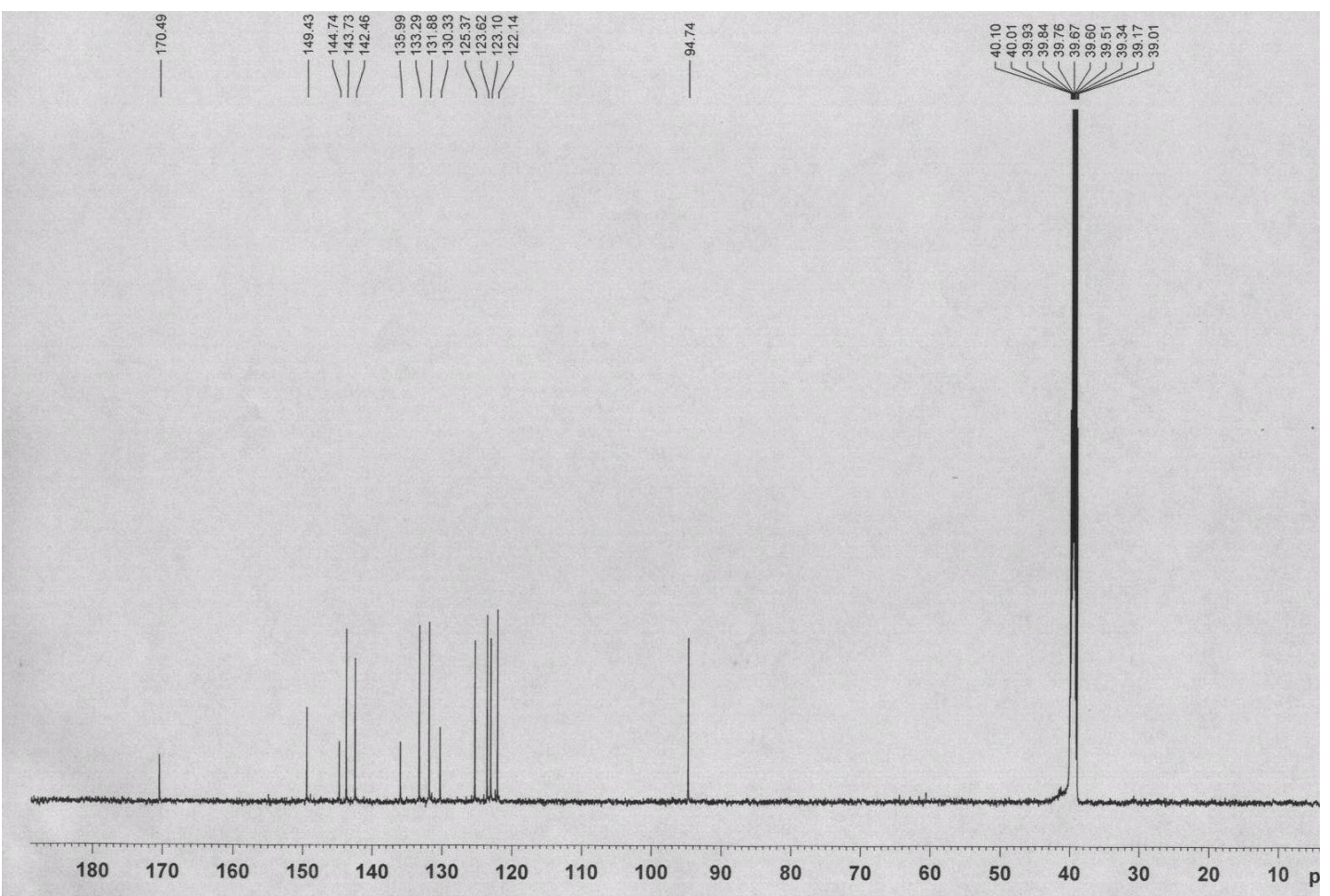

${ }^{1}$ H-NMR Spectra of Compound 2

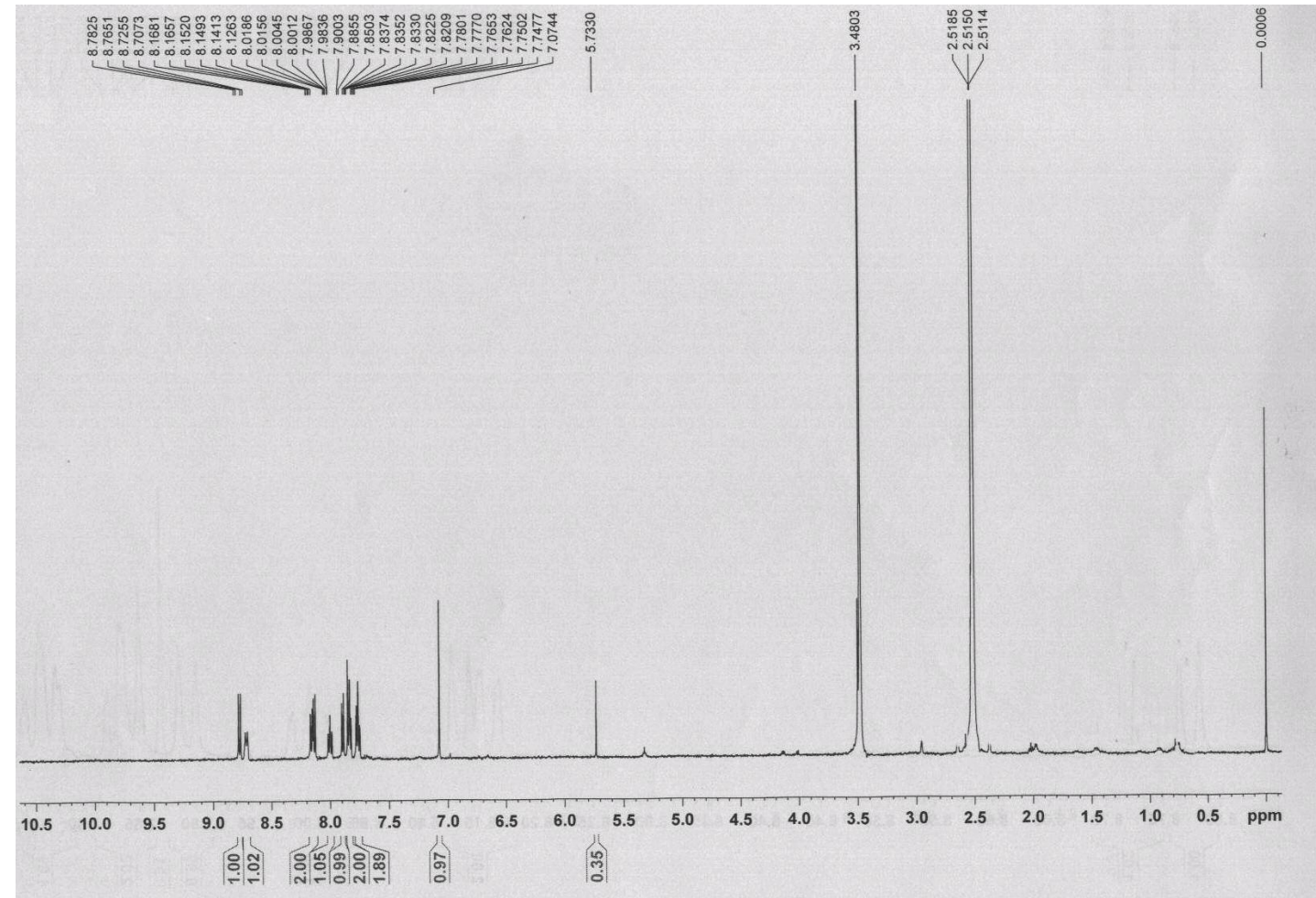




\section{${ }^{13}$ C-NMR spectra of Compound 2}

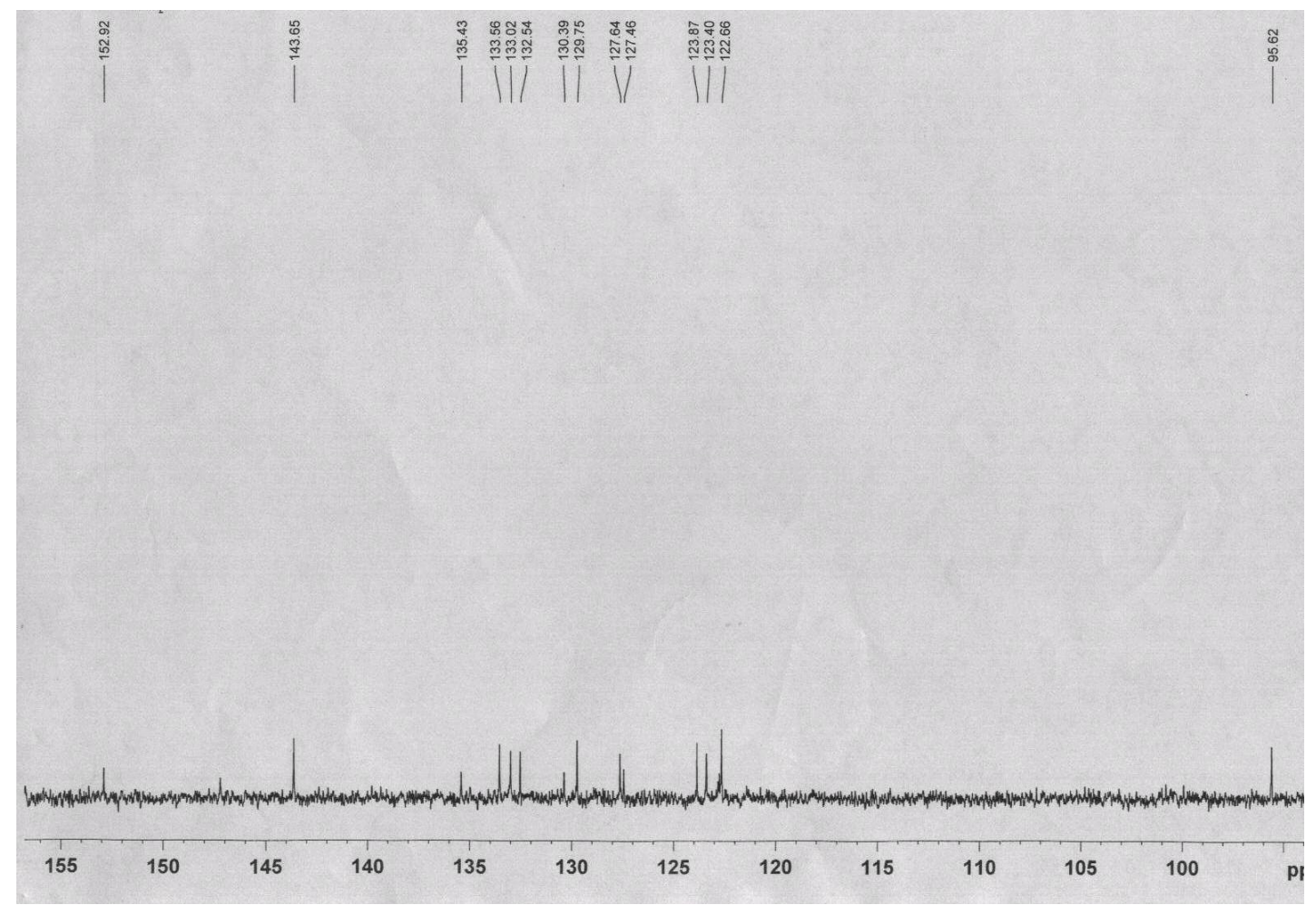

Symbolic Z-matrix for compound 1

\begin{tabular}{|c|c|c|c|c|c|c|}
\hline \multicolumn{7}{|l|}{3} \\
\hline C 1 & 1 B1 & & & & & \\
\hline C 2 & 2 B2 & 1 & A1 & & & \\
\hline $\mathrm{C}$ & B3 & 1 & A2 & $3 \mathrm{I}$ & D1 & 0 \\
\hline C 3 & B4 & 2 & A3 & 1 & D2 & 0 \\
\hline C 3 & B5 & 2 & A4 & $1 \mathrm{I}$ & D3 & 0 \\
\hline C 6 & B6 & 3 & A5 & $2 \mathrm{I}$ & D4 & 0 \\
\hline C 7 & B7 & 6 & A6 & $3 \mathrm{I}$ & D5 & 0 \\
\hline C 6 & B8 & $3 t$ & A7 & 2 & D6 & 0 \\
\hline C 7 & B9 & 6 & A8 & 3 & D7 & 0 \\
\hline C 4 & B10 & $2 t$ & A9 & $1 \mathrm{I}$ & D8 & 0 \\
\hline C 8 & $8 \quad$ B11 & 7 & A10 & 6 & D9 & 0 \\
\hline C 8 & B12 & 7 & A11 & 6 & D10 & 0 \\
\hline C 2 & $2 \quad$ B13 & 1 & A12 & 6 & D11 & 0 \\
\hline C 1 & $3 \quad$ B14 & 8 & A13 & 7 & D12 & 0 \\
\hline F 1 & B15 & 2 & A14 & 14 & D13 & 0 \\
\hline F 1 & B16 & 2 & A15 & 14 & D14 & 0 \\
\hline H 1 & $\begin{array}{ll}10 & \text { B17 }\end{array}$ & 7 & A16 & 6 & D15 & 0 \\
\hline $\mathrm{H}$ & $9 \quad \mathrm{~B} 18$ & 6 & A17 & 3 & D16 & 0 \\
\hline $\mathrm{H} 2$ & $2 \quad$ B19 & 1 & A18 & 6 & D17 & 0 \\
\hline H 5 & B20 & 3 & A19 & 2 & D18 & 0 \\
\hline H 1 & $5 \quad \mathrm{~B} 21$ & 13 & A20 & 8 & D19 & 0 \\
\hline $\mathrm{H}$ & $4 \quad$ B22 & 2 & A21 & 1 & D20 & 0 \\
\hline $\mathrm{H}$ & $14 \mathrm{~B} 23$ & 2 & A22 & 1 & D21 & 0 \\
\hline $\mathrm{H}$ & $13 \quad$ B24 & 8 & A23 & 7 & D22 & 0 \\
\hline $\mathrm{H}$ & $11 \quad$ B25 & 4 & A24 & 2 & D23 & 0 \\
\hline $\mathrm{N}$ & $6 \quad$ B26 & 3 & A25 & 2 & D24 & 0 \\
\hline $\mathrm{N}$ & $2 \quad$ B27 & 1 & A26 & & $\begin{array}{ll}7 & \mathrm{D} 25\end{array}$ & 0 \\
\hline O 1 & $12 \quad$ B28 & 8 & A27 & 7 & $\mathrm{D} 26$ & 0 \\
\hline & Variables: & & & & & \\
\hline B1 & & & 52198 & & & \\
\hline B2 & & 2.3 & & & & \\
\hline B3 & & & 40011 & & & \\
\hline
\end{tabular}




\begin{tabular}{|c|c|}
\hline B4 & 6.04938 \\
\hline B5 & 2.44134 \\
\hline B6 & 1.47357 \\
\hline B7 & 1.4056 \\
\hline B8 & 1.36671 \\
\hline B9 & 1.39438 \\
\hline B10 & 1.38551 \\
\hline B11 & 1.4892 \\
\hline B12 & 1.38926 \\
\hline B13 & 1.38238 \\
\hline B14 & 1.40326 \\
\hline B15 & 1.42649 \\
\hline B16 & 1.41757 \\
\hline B17 & 1.08515 \\
\hline B18 & 1.08167 \\
\hline B19 & 1.08089 \\
\hline B20 & 1.08506 \\
\hline B21 & 1.08488 \\
\hline B22 & 1.08448 \\
\hline B23 & 1.08207 \\
\hline B24 & 1.08446 \\
\hline B25 & 1.08333 \\
\hline B26 & 1.38748 \\
\hline B27 & 1.36259 \\
\hline B28 & 1.23533 \\
\hline A1 & 64.83558 \\
\hline $\mathrm{A} 2$ & 125.72043 \\
\hline A3 & 138.7243 \\
\hline A4 & 119.74661 \\
\hline A5 & 158.75054 \\
\hline A6 & 107.12533 \\
\hline A7 & 29.99199 \\
\hline A8 & 132.16518 \\
\hline A9 & 89.25593 \\
\hline A10 & 108.47479 \\
\hline A11 & 121.67701 \\
\hline A12 & 156.53387 \\
\hline A13 & 117.81601 \\
\hline A14 & 97.04097 \\
\hline A 15 & 84.58997 \\
\hline A16 & 121.69836 \\
\hline A17 & 120.32072 \\
\hline A18 & 80.01631 \\
\hline A19 & 131.92508 \\
\hline A 20 & 119.78523 \\
\hline A21 & 150.60269 \\
\hline A22 & 119.97451 \\
\hline A 23 & 120.56986 \\
\hline A24 & 121.08151 \\
\hline A 25 & 92.74742 \\
\hline A26 & 35.21532 \\
\hline A27 & 128.14882 \\
\hline D1 & 5.14349 \\
\hline D2 & -14.92525 \\
\hline D3 & -10.05614 \\
\hline D4 & -167.20173 \\
\hline D5 & 169.88693 \\
\hline D6 & -179.82056 \\
\hline D7 & -9.64609 \\
\hline D8 & -5.35869 \\
\hline D9 & 0.35093 \\
\hline D10 & -179.50832 \\
\hline D11 & 19.97225 \\
\hline D12 & -0.2424 \\
\hline D13 & -97.27607 \\
\hline D14 & 152.64623 \\
\hline D15 & -0.4868 \\
\hline D16 & 176.3731 \\
\hline D17 & -167.73833 \\
\hline
\end{tabular}




$\begin{array}{lc}\text { D18 } & -172.91118 \\ \text { D19 } & -179.80822 \\ \text { D20 } & 174.65507 \\ \text { D21 } & 170.52688 \\ \text { D22 } & 179.89854 \\ \text { D23 } & -179.33379 \\ \text { D24 } & 3.34573 \\ \text { D25 } & 20.41973 \\ \text { D26 } & 179.04695\end{array}$

Symbolic Z-matrix for compound 2 B

C 11 B1

$\begin{array}{lllll}\mathrm{C} & 1 & \mathrm{~B} 2 & 2 & \mathrm{~A} 1\end{array}$

$\begin{array}{llllllll}\mathrm{C} & 1 & \mathrm{~B} 3 & 3 & \mathrm{~A} 2 & 2 & \mathrm{D} 1 & 0\end{array}$

$\begin{array}{llllllll}\text { C } & 3 & \text { B4 } & 1 & \text { A3 } & 4 & \text { D2 } & 0\end{array}$

$\begin{array}{llllllll}\mathrm{C} & 3 & \mathrm{~B} 5 & 1 & \mathrm{~A} 4 & 4 & \mathrm{D} 3 & 0\end{array}$

$\begin{array}{llllllll}\mathrm{C} & 2 & \mathrm{~B} 6 & 1 & \mathrm{~A} 5 & 6 & \mathrm{D} 4 & 0\end{array}$

$\begin{array}{llllllll}\mathrm{C} & 4 & \mathrm{~B} 7 & 1 & \mathrm{~A} 6 & 6 & \mathrm{D} 5 & 0\end{array}$

$\begin{array}{llllllll}\text { C } & 8 & \text { B8 } & 4 & \text { A7 } & 1 & \text { D6 } & 0\end{array}$

$\begin{array}{llllllll}\text { C } & 9 & \text { B9 } & 8 & \text { A8 } & 4 & \text { D7 } & 0\end{array}$

$\begin{array}{llllllll}\mathrm{C} & 8 & \mathrm{~B} 10 & 4 & \mathrm{~A} 9 & 1 & \mathrm{D} 8 & 0\end{array}$

$\begin{array}{lllllll}\text { C } 9 & \text { B11 } & 8 & \text { A10 } & 4 & \text { D9 } & 0\end{array}$

$\begin{array}{llllllll}\mathrm{C} & 5 & \mathrm{~B} 12 & 3 & \mathrm{~A} 11 & 1 & \mathrm{D} 10 & 0\end{array}$

$\begin{array}{llllllll}\text { C } & 5 & \text { B13 } & 3 & \text { A12 } & 1 & \text { D11 } & 0\end{array}$

$\begin{array}{llllllll}\mathrm{C} & 14 & \mathrm{~B} 14 & 5 & \mathrm{~A} 13 & 3 & \mathrm{D} 12 & 0\end{array}$

$\begin{array}{llllllll}\text { C } & 10 & \text { B15 } & 9 & \text { A14 } & 8 & \text { D13 } & 0\end{array}$

$\begin{array}{llllllll}\mathrm{C} & 10 & \mathrm{~B} 16 & 9 & \mathrm{~A} 15 & 8 & \mathrm{D} 14 & 0\end{array}$

$\begin{array}{llllllll}\mathrm{C} & 13 & \mathrm{~B} 17 & 5 & \mathrm{~A} 16 & 3 & \mathrm{D} 15 & 0\end{array}$

$\begin{array}{llllllll}\mathrm{C} & 3 & \mathrm{~B} 18 & 1 & \mathrm{~A} 17 & 8 & \mathrm{D} 16 & 0\end{array}$

$\begin{array}{llllllll}\mathrm{F} & 1 & \mathrm{~B} 19 & 8 & \mathrm{~A} 18 & 4 & \mathrm{D} 17 & 0\end{array}$

$\begin{array}{llllllll}\mathrm{F} & 1 & \mathrm{~B} 20 & 8 & \mathrm{~A} 19 & 4 & \mathrm{D} 18 & 0\end{array}$

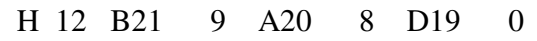

$\begin{array}{llllllll}\mathrm{H} & 11 & \mathrm{~B} 22 & 8 & \mathrm{~A} 21 & 4 & \mathrm{D} 20 & 0\end{array}$

$\begin{array}{llllllll}\mathrm{H} & 18 & \mathrm{~B} 23 & 13 & \mathrm{~A} 22 & 5 & \mathrm{D} 21 & 0\end{array}$

$\begin{array}{llllllll}\mathrm{H} & 2 & \mathrm{~B} 24 & 1 & \mathrm{~A} 23 & 8 & \mathrm{D} 22 & 0\end{array}$

$\begin{array}{llllllll}\mathrm{H} & 7 & \mathrm{~B} 25 & 2 & \mathrm{~A} 24 & 1 & \mathrm{D} 23 & 0\end{array}$

$\begin{array}{llllllll}\mathrm{H} & 19 & \mathrm{~B} 26 & 3 & \mathrm{~A} 25 & 1 & \mathrm{D} 24 & 0\end{array}$

$\begin{array}{llllllll}\mathrm{H} & 3 & \mathrm{~B} 27 & 1 & \mathrm{~A} 26 & 8 & \mathrm{D} 25 & 0\end{array}$

$\begin{array}{llllllll}\mathrm{H} & 17 & \mathrm{~B} 28 & 10 & \mathrm{~A} 27 & 9 & \mathrm{D} 26 & 0\end{array}$

$\begin{array}{llllllll}\mathrm{H} & 15 & \mathrm{~B} 29 & 14 & \mathrm{~A} 28 & 5 & \mathrm{D} 27 & 0\end{array}$

$\begin{array}{llllllll}\mathrm{H} & 14 & \mathrm{~B} 30 & 5 & \mathrm{~A} 29 & 3 & \mathrm{D} 28 & 0\end{array}$

$\begin{array}{llllllll}\mathrm{H} & 13 & \mathrm{~B} 31 & 5 & \mathrm{~A} 30 & 3 & \mathrm{D} 29 & 0\end{array}$

$\begin{array}{llllllll}\mathrm{N} & 4 & \mathrm{~B} 32 & 1 & \mathrm{~A} 31 & 8 & \mathrm{D} 30 & 0\end{array}$

$\begin{array}{llllllll}\mathrm{N} & 8 & \mathrm{~B} 33 & 4 & \mathrm{~A} 32 & 1 & \mathrm{D} 31 & 0\end{array}$

$\begin{array}{llllllll}\mathrm{O} & 16 & \mathrm{~B} 34 & 10 & \mathrm{~A} 33 & 9 & \mathrm{D} 32 & 0\end{array}$ Variables:

B1

B3 2.61356

B4 2.45088

B5 1.41813

B6 1.40469

B7 2.44282

B8 $\quad 1.47322$

B9 $\quad 1.40564$

B10 1.36556

B11 1.39416

B12 1.41809

B13 1.42427

B14 1.36596

B15 1.48985

B16 1.38896

B17 1.38095

B18 1.38482

B19 1.42566

B20 1.42575

B21 1.08512

B22 1.08141

B23 1.08444 


\begin{tabular}{|c|c|}
\hline B24 & 1.08504 \\
\hline B25 & 1.0849 \\
\hline B26 & 1.08465 \\
\hline B27 & 1.07769 \\
\hline B28 & 1.08444 \\
\hline B29 & 1.08294 \\
\hline B30 & 1.08546 \\
\hline B31 & 1.08573 \\
\hline B32 & 1.36757 \\
\hline B33 & 1.37974 \\
\hline B34 & 1.23481 \\
\hline A1 & 159.8145 \\
\hline $\mathrm{A} 2$ & 84.40713 \\
\hline A3 & 93.81857 \\
\hline A4 & 63.02753 \\
\hline A5 & 83.72654 \\
\hline A6 & 61.00854 \\
\hline A7 & 159.43679 \\
\hline A8 & 106.97492 \\
\hline A9 & 29.78664 \\
\hline A10 & 132.26249 \\
\hline A11 & 89.30253 \\
\hline A12 & 149.09661 \\
\hline A13 & 120.1978 \\
\hline A14 & 108.41676 \\
\hline A15 & 121.66313 \\
\hline A16 & 120.58424 \\
\hline A17 & 177.04402 \\
\hline A18 & 121.35608 \\
\hline A19 & 121.2357 \\
\hline A20 & 121.70194 \\
\hline A21 & 120.33938 \\
\hline A22 & 120.50941 \\
\hline A23 & 156.83625 \\
\hline A24 & 119.45937 \\
\hline A25 & 118.91375 \\
\hline A26 & 57.12284 \\
\hline A27 & 120.59451 \\
\hline A28 & 121.40192 \\
\hline A 29 & 119.0372 \\
\hline A30 & 118.52622 \\
\hline A 31 & 31.64981 \\
\hline A32 & 92.06878 \\
\hline A33 & 128.1733 \\
\hline D1 & 0.10773 \\
\hline D2 & -0.00857 \\
\hline D3 & 0 \\
\hline D4 & -179.86599 \\
\hline D5 & -179.9607 \\
\hline D6 & 179.96672 \\
\hline D7 & -179.88467 \\
\hline D8 & -179.92758 \\
\hline D9 & 0.11612 \\
\hline D10 & -179.98704 \\
\hline D11 & 0.00935 \\
\hline D12 & -0.00519 \\
\hline D13 & -0.02242 \\
\hline D14 & 179.98938 \\
\hline D15 & 0 \\
\hline D16 & -179.76578 \\
\hline D17 & 106.02163 \\
\hline D18 & -105.91885 \\
\hline D19 & 0 \\
\hline D20 & -179.97193 \\
\hline D21 & 179.99674 \\
\hline $\mathrm{D} 22$ & 0.13341 \\
\hline D23 & -179.98093 \\
\hline D24 & -0.29686 \\
\hline D25 & 179.90908 \\
\hline
\end{tabular}




$\begin{array}{cc}\text { D26 } & -180 . \\ \text { D27 } & -180 . \\ \text { D28 } & 180 . \\ \text { D29 } & -180 . \\ \text { D30 } & 179.93521 \\ \text { D31 } & 0.05684 \\ \text { D32 } & -180 .\end{array}$

\section{Reference}

[1] Norman, M. H.; Minick D. J.; Rigdon, G. C. J. Med. Chem. 1996, 39, 149-157

[2] Chapman, J. M.; Cocolas, G. H.; Hall, I. H. J. Med Chem. 1983, 26, 243-246.

[3] Bergstrom, F.; Mikhalyoy, I.; Hagglof, P.; Wortmann, R.; Ny, T.; Johansson, L. J. Am. Chem. Soc. 2002, 124, $196-204$. 